\title{
Bitrate Requirements of Non-Panoramic VR Remote Rendering
}

\author{
Viktor Kelkkanen \\ Blekinge Institute of Technology \\ Karlskrona, Sweden \\ viktor.kelkkanen@bth.se
}

\author{
Markus Fiedler \\ Blekinge Institute of Technology \\ Karlshamn, Sweden \\ markus.fiedler@bth.se
}

\author{
David Lindero \\ Ericsson \\ Luleå, Sweden \\ david.lindero@ericsson.com
}

ABSTRACT

This paper shows the impact of bitrate settings on objective quality measures when streaming non-panoramic remote-rendered Virtual Reality (VR) images. Non-panoramic here refers to the images that are rendered and sent across the network, they only cover the viewport of each eye, respectively.

To determine the required bitrate of remote rendering for VR, we use a server that renders a 3D-scene, encodes the resulting images using the NVENC H.264 codec and transmits them to the client across a network. The client decodes the images and displays them in the VR headset. Objective full-reference quality measures are taken by comparing the image before encoding on the server to the same image after it has been decoded on the client. By altering the average bitrate setting of the encoder, we obtain objective quality scores as functions of bitrates. Furthermore, we study the impact of headset rotation speeds, since this will also have a large effect on image quality.

We determine an upper and lower bitrate limit based on headset rotation speeds. The lower limit is based on a speed close to the average human peak head-movement speed, $360 \%$ s. The upper limit is based on maximal peaks of $1080 \%$ s. Depending on the expected rotation speeds of the specific application, we determine that a total of $20-38 \mathrm{Mbps}$ should be used at resolution 2160×1200@90 fps, and $22-42 \mathrm{Mbps}$ at $2560 \times 1440 @ 60 \mathrm{fps}$. The recommendations are given with the assumption that the image is split in two and streamed in parallel, since this is how the tested prototype operates.

\section{CCS CONCEPTS}

- Networks $\rightarrow$ Cloud computing; Network measurement; • Applied computing $\rightarrow$ Computer games; $\bullet$ Computing methodologies $\rightarrow$ Virtual reality.

\section{KEYWORDS}

remote rendering, game streaming, low-latency, 6-DOF, SSIM, VMAF

\section{ACM Reference Format:}

Viktor Kelkkanen, Markus Fiedler, and David Lindero. 2020. Bitrate Requirements of Non-Panoramic VR Remote Rendering. In Proceedings of the 28th ACM International Conference on Multimedia (MM '20), October 12-16, 2020, Seattle, WA, USA. ACM, New York, NY, USA, 8 pages. https: //doi.org/10.1145/3394171.3413681

Permission to make digital or hard copies of all or part of this work for personal or classroom use is granted without fee provided that copies are not made or distributed for profit or commercial advantage and that copies bear this notice and the full citation on the first page. Copyrights for components of this work owned by others than the author(s) must be honored. Abstracting with credit is permitted. To copy otherwise, or republish, to post on servers or to redistribute to lists, requires prior specific permission and/or a fee. Request permissions from permissions@acm.org.

MM '20, October 12-16, 2020, Seattle, WA, USA

(1) 2020 Copyright held by the owner/author(s). Publication rights licensed to ACM. ACM ISBN 978-1-4503-7988-5/20/10 ..\$15.00

https://doi.org/10.1145/3394171.3413681

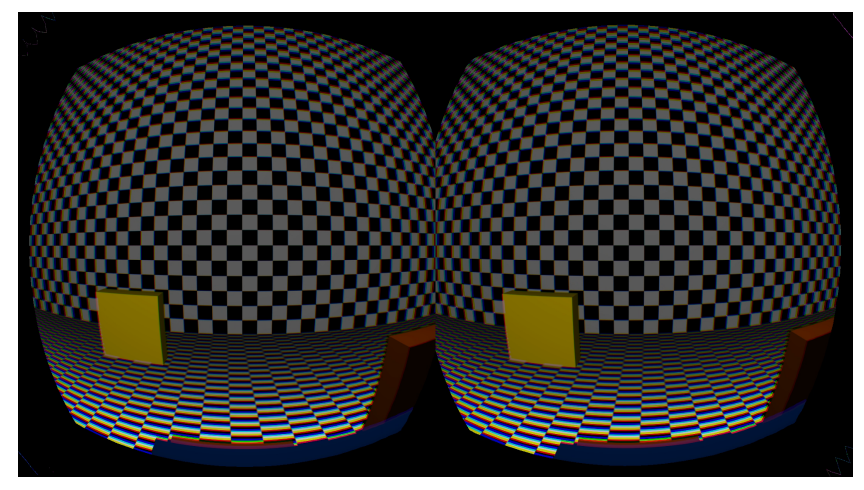

Figure 1: Example of the non-panoramic view and 3D scene that is rendered once per eye and streamed each frame.

\section{INTRODUCTION}

Remote rendering for VR is commonly done by using panorama images that contain a 360-degree-view from the current position of the Head Mounted Display (HMD). Such panorama techniques may reduce rotational latency and provide a more seamless experience than techniques that only render the current viewport. This is due to the fact that the panorama image has information of every viewing direction, and can therefore easily select a new view depending on the look-vector of the HMD on the local device. However, as those images cover a much larger area, they require more data and rendering power to be able to produce an image quality that matches that of the non-panoramic method. The technique is also not ideal for very interactive scenes, i.e. games, because any interaction with the world must immediately be shown anyway. This must be done either by downloading a new image or rendering it on the client. Rendering new panorama images on the server in real time can also be very time consuming due to the stitching process and need for six cameras. This is why we see potential in the simpler method where just the current viewport that is seen by the user is rendered, as would be the case in local rendering. Figure 1 shows an example of the non-panoramic view used in this work.

The method of rendering just the viewport is commonly used in short-range remote rendering for VR, for example when enabling wireless streaming to HMDs in the same room as the rendering computer, e.g. when using TPCAST [17]. It may be possible to expand this to networks with longer range in the future. It would put immense requirements on those networks though, because new images must constantly be downloaded at a high framerate to cover the current view-direction of the user. Naturally, many problems arise from this task, perhaps most critically are the ones related to network latency and codec time consumption. Those issues will be covered in a different publication though, in this work we focus 
on the issue of bandwidth requirements. The bandwidth may be more limited than on aAN with a WiGig connection or similar. Thus, we need to determine in depth the bitrate requirements of this type of streaming, which is the scope of this work. We determine bitrate requirements by using an existing prototype, developed inhouse, that remotely renders a VR scene once per eye at a common HMD resolution, 1080×1200@90 fps, and mobile phone resolution 1280×1440@60 fps. From now on, the unit fps will be omitted when describing a resolution and its framerate.

The remainder of the paper is structured as follows: Section 2 presents previous work that is related to VR streaming and required bitrates, Section 3 describes the methods we have used to determine the bitrate requirements, Section 4 shows results from experiments with the prototype, Section 5 concludes the work and presents our most important findings, Section 6 discusses potential future work.

\section{RELATED WORK}

Related work can be divided into three areas with a decreasing level of relevance: First, other studies of remote rendering solutions for non-panoramic VR, second, studies of remote rendering or video streaming of 360 panoramic images for VR, and finally, solutions for cloud gaming.

\subsection{Non-Panoramic VR Remote Rendering}

We have found two public works where authors have created systems for non-panoramic VR streaming and put them to the test $[9,20]$. [20] does not bring up bitrate, and [9] leaves room for deeper investigation into bitrate requirements. In [9], authors present results related to bitrate in the form of a maximum frame size setting that is set to slightly more than $200 \mathrm{~KB}$ to reach a Structural SIMilarity (SSIM) [21] above 0.98. This number, 0.98, is used as the limit for a visually lossless experience, the authors base this on related previous work [1]. It is not clear what a maximum frame size means in terms of overall bitrate requirements. To our understanding, $200 \mathrm{~KB}$ per frame would in the worst case be equal to $200 \times 1024 \times 8 \times 90=147 \mathrm{Mbps}$, but is likely lower on average. Their solution runs at $2160 \times 1200 @ 90$ at this bitrate.

\section{$2.2360 \mathrm{VR}$}

The authors of [8] develop a solution for streaming of $360 \mathrm{VR}$ by using pre-rendered panorama images. This solution requires on average 132 Mbps when running 2560×1440@60 and reaches a maximum average SSIM of 0.944 when tested with different scenes. The solution is not optimal for interactive scenes, as it requires pre-rendering of panoramas at every possible user position.

Another work in 360 VR studies the impact of bitrate on MOS when encoding with HEVC (H.265) at 3840×2160@30 [15]. Results show that the most cost-efficient bitrate in their case was $8 \mathrm{Mbps}$, the MOS at this bitrate was around 4.25 on a 5 -grade scale. Raising the bitrate to $15 \mathrm{Mbps}$ would increase MOS only slightly to around 4.4 .

\subsection{Cloud Gaming}

Related work on a streaming solution for cloud gaming using collaborative rendering [1] shows a requirement of less than $5000 \mathrm{Kbps}$ to achieve an SSIM above 0.98 . The solution is intended for mobile phones and works at a resolution of $1280 \times 720 @ 60$.

A work on foveated video encoding for cloud gaming on mobile phones present a maximum bitrate of $10 \mathrm{Mbps}$ that results in a Mean Opinion Score (MOS) of 69 on a scale of $0-100$ [6]. 1920×1080@50 is reported as the used resolution.

\section{METHOD}

In order to determine the image quality as function of bitrate and HMD movement, we conduct experiments with a VR remote rendering prototype where one parameter is altered at a time and the impact on two objective quality estimators are recorded, SSIM and Video Multi-Method Assessment Fusion (VMAF) [10].

\subsection{Software}

An existing solution for non-panoramic VR remote rendering is used. This solution was developed in-house and consists of a server that renders a 3D-scene by using $\mathrm{C}++$ and OpenGL, and a client that displays received images. The scene is rendered once per eye and thus creates two textures that are encoded, transmitted and decoded in parallel. When calculating quality scores, we only use the left-eye image, this is because the two images are very similar and never merged in the program. An alternative would be to calculate the scores for both images, and report the average, or artificially merge the two into one image for comparison. Combining the two would double the size of recorded data though, and not make much difference in the analysis according to our observations. Therefore, we only use one of the images to determine the scores. As previously stated, the images are encoded in parallel, this happens in two separate hardware encoders that use the exact same settings, including bitrate. This means that the total bitrate required for the system is two times that of the individual encoder bitrate setting. When a bitrate is presented in this document, it refers to the bitrate of one individual eye, unless specifically stated otherwise. It should thus be doubled to acquire the total bitrate of the system.

3.1.1 Codec. The Nvidia Video Codec SDK (NVENC and NVDEC) [12] is used in $\mathrm{C}++$ for encoding and decoding. Speed is the most important priority in our use-case since VR requires a very low latency. To our knowledge, since we test on Nvidia hardware, the Nvidia Video Codec is the fastest alternative available to us at this time, which is why it was chosen. All testing in this work is done with a GTX 1080 as encoder and a GTX 950 as decoder.

We use raw H.264 with an infinite group of pictures, starting with one I-frame followed by an infinite amount of P-frames. This is possible due to the design of the prototype, which is able to operate without data loss. Note that a detailed description of the prototype is outside the scope of this work, but we provide here some details that are relevant to the bitrate. YUV420p (NV12) is used as video surface format and the rate control mode is set to constant bitrate high quality setting (NV_ENC_PARAMS_RC_CBR_LOWDELAY_HQ). Two resolutions are tested, $1080 \times 1200$ and $1280 \times 1440$ per eye at framerates 90 and $60 \mathrm{fps}$, respectively. Other than these settings, the codec is based on the C++ samples from the Nvidia Video Codec SDK [12], "EncLowLatency.cpp" and "DecLowLatency.cpp", but with added OpenGL and CUDA functionality for immediate streaming. Please refer to Table 1 for detailed NVENC settings. 
Table 1: NVENC Settings

\begin{tabular}{|c|c|}
\hline Parameter & Value \\
\hline rateControlMode & $\begin{array}{l}\text { NV_ENC_PARAMS_RC_CBR } \\
\text { _LOWDELAY_HQ }\end{array}$ \\
\hline averageBitRate & Varying \\
\hline vbvBufferSize & averageBitRate * (1 / frameRate) \\
\hline vbvInitialDelay & vbvBufferSize \\
\hline zeroReorderDelay & 1 \\
\hline gopLength & NVENC_INFINITE_GOPLENGTH \\
\hline frameIntervalP & 1 \\
\hline
\end{tabular}

H.264 was chosen over H.265 due to the reported encoding speed by NVIDIA [12]. The H.264 implementation is $\sim 30 \%$ faster on average at $1080 \mathrm{p}$, making it preferable in this case. We also decided to use two separate streams to enable parallel encoding using two NVENC encoders found on certain graphics cards, making the encoding faster than just using one big frame for both views.

3.1.2 Quality Estimation. To calculate a per-frame quality estimate, we capture the image of a specific index at both the server before encoding, and on the client after decoding. Each image is saved to disk by using the lossless format PNG and together converted to a video file for each test sequence later. This is done with FFmpeg [2] using its raw video codec, YUV420p pixel format and YUV container as storage.

SSIM and VMAF are calculated by comparing the resulting two YUV-videos by using the VMAF Development Kit (VDK) by Netflix to calculate VMAF [10], and FFmpeg to calculate SSIM [3]. The outputs from both programs contain per-frame calculations of SSIM or VMAF and can be used to show the instantaneous scores of each frame in the video.

VMAF is included in the analysis since it provides a better match to the subjective video quality level than the commonly used SSIM [5]. The VR image size used per eye in this work, $1080 \times 1200$, is not a perfect match to the $1920 \times 1080$ resolution that the VMAF model has been optimized for, but it seems the model can handle other formats and VR-like content in a good way according to [13]. The authors of that work conclude that the VMAF model can be used with $3850 \times 1920360$ VR images with an HMD as display. We use the same model in our work, version 0.6 .1 , which was originally trained for 1080p HDTV in a living-room-like environment [11].

SSIM is still also presented in this work since it is very common in VR image quality analysis, and this makes it more straightforward to compare the results with other studies.

\subsection{Image Content}

The content of the image is an important consideration due to its large impact on resulting quality. A complex scene with e.g. heavy movement is more difficult for the encoder to encode efficiently, when compared to a scene that is perfectly still or only contains little movement. Increased complexity will generally result in a higher bitrate requirement to maintain the same quality level. In order to determine robust bitrate requirements, it is thus important that the tested scene is sufficiently complex to account for unknown

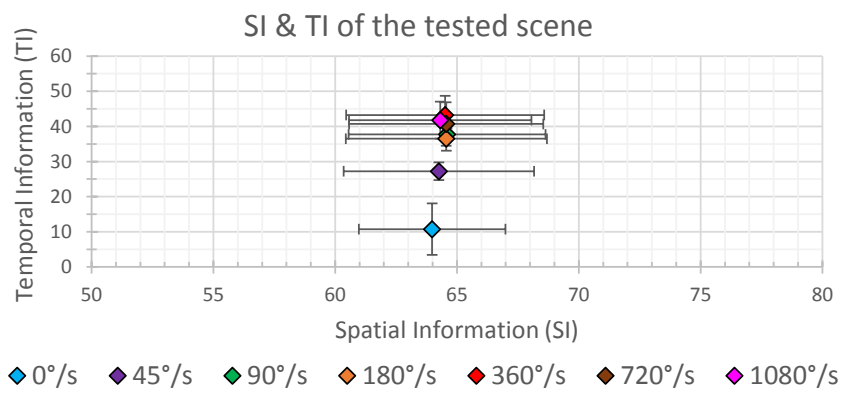

Figure 2: SI and TI scores of the checkerboard scene at various rotations speeds recorded at $1080 \times 1200 @ 90$. The bar values of each measurement indicates the minimum and maximum SI and TI, respectively.

complexity as it may appear in real scenarios. Not only is it important to account for complex imagery, but one must also account for the movement in pixels that is produced by the user moving the HMD. This movement may indeed also increase complexity and thus the bitrate requirement.

3.2.1 3D Scene. We use a scene with a black and white checkerboard background that contains colored cubes that rotate around the user at a fixed distance and speed, see Figure 1. To test for fast-moving objects, the cubes rotate very fast around the camera, they complete one revolution around the camera approximately every 1.5 seconds, depending on the framerate. User head rotations are simulated by rotating the camera at various speeds in software. To test whether this scene is a valid substitute for a real game, we calculate its Temporal Information (TI) and Spatial Information (SI) [7] at various head rotation speeds, see Figure 2, and compare them to existing references. In the ITU-T Recommendation P.910 [7], most tested scenes seem to cluster around roughly $\mathrm{SI} \in[50,100]$ and TI $\in[0,40]$. In related work on cloud gaming [16], we see roughly SI $\in[65,85]$ and TI $\in[30,80]$ from recordings of commercial games. One example of such a game scores very close to $\mathrm{SI}=65$ in five tests, with a TI $\in[35,55]$. Thus, our measured values SI $\in[60,69]$ and $\mathrm{TI} \in[3,49]$ indicate a fairly similar categorization. The SI and TI of most of our test-sequences are gathered in one cluster, with two outliers related to the 0 and $45 \%$ rotations. The difference in TI indicates that any movement of $90 \% \mathrm{~s}$ and above will not increase the TI but make it remain at a relatively high value of around 40.

3.2.2 Head Movement. In order to emulate user movements in a reproducible way, we apply rotations to the VR camera in software, with an angle around the vertical Y-axis (yaw). In experiments with continuous rotation, the viewing angle of the VR camera ranges between $-180^{\circ}-180^{\circ}$ and changes at a variable rate in degrees per second. The user rotation speeds in a real scenario will depend on the application. A game may for example present most of its content in front of the user, requiring little rotation, or it may require them to look around often and quickly due to some game rule. Determining in depth the maximum and average rotation speeds of users in VR is left for future work. We performed a small in-house user test in this work though, which is described in the next section. By recording 
rotations while playing a VR game, we obtained realistic human head movements. This data is used in further testing and to verify that assumptions are realistic. In experiments on objective quality, we test rotations from $0-1080^{\circ} / \mathrm{s}$ and assume this covers the realistic range of rotation speeds of human heads. We have selected $1080^{\circ} / \mathrm{s}$ as the maximum turn rate in this work due to peak rotation speeds of the human head allegedly reaching around $800 \%$ [4]. We use $360 \%$ as the average rotation speed and as a minimum requirement. This is based on a medical study unrelated to VR that determined that a control group of an experiment regarding neck injury would rotate their heads on average at $348 \pm 92^{\circ} / \mathrm{s}$ [14].

3.2.3 In-House User Test. To confirm that the assumptions of a $360 \%$ s average and $1080 \%$ s maximum based on literature are not too far off from reality, we conduct one user test with a person involved in the project. This test records the rotation of the HMD during a VR gaming session by using an external process that polls HMD pose data using the OpenVR API by Valve [18]. This process polls the pose approximately every $1 / 90$ th of a second, Euler angles are then extracted from the pose matrix and saved to disk, from which we calculate the rotation deltas of each frame. The game played in the test is a mini-game called Longbow that is part of a compilation of games called The Lab by Valve [19]. In Longbow, the player defends a castle from attackers by shooting them using bow and arrow, this requires a moderate amount of head-movement.

From the recording on user test head-rotations, we obtain Figure 3, which shows the orientation of the HMD during each frame of the test. We note that the dominant rotation is yaw, which is the rotation around the vertical axis, i.e. looking around to the sides left and right. The up-and-down rotation, the pitch, ranges between $45^{\circ}$ and $-90^{\circ}$. Pitch is used in the game to aim down the castle wall towards attackers and upwards to the sky in order to shoot balloons. Roll is naturally not used much due to the human anatomy but will show up to small degrees when tilting the head to the side.

The Complementary Cumulative Distribution Functions (CCDF) of the rotation speeds are shown in Figure 4. This figure is based on 86886 samples recorded during the user test and shows how common the different rotation speeds are. For example, we find that $10 \%$ of rotations are faster than $60 \%, 1 \%$ of rotations are faster than $230 \%$ s and $0.1 \%$ of rotations are faster than $315 \%$ s and so on, all in terms of yaw which is the fastest. The fastest recorded rotation from the test is $412 \%$ s. We conclude that our assumption that at least $360^{\circ} / \mathrm{s}$ should be supported at all times is reasonable though might be slightly on the lower end.

Another test was conducted during which the user tried rotating their head as fast as possible. This showed peak speeds of 1000 $1300 \%$ s. The fastest of these rotations did not seem practical for games though as they involved moving the whole body simultaneously as the head and were overall on the brink of being painful. We therefore find that $1080^{\circ} \mathrm{s}$ is acceptable as an upper limit.

\subsection{Modelling}

Upon obtaining SSIM and VMAF values for given rotation speeds $V$ (in $\%$ ) and bitrates $R$ (in Mbps), we analyse them for relationships

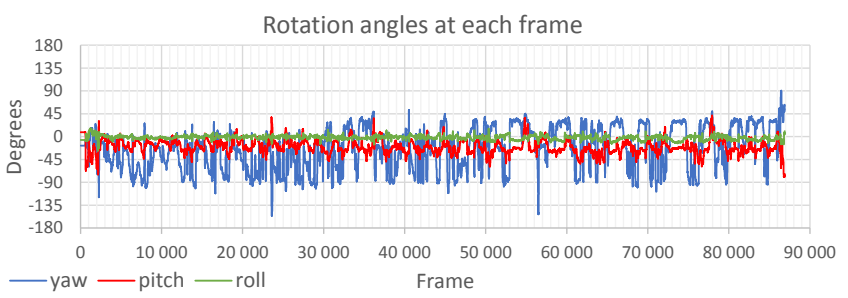

Figure 3: Rotations during user test.

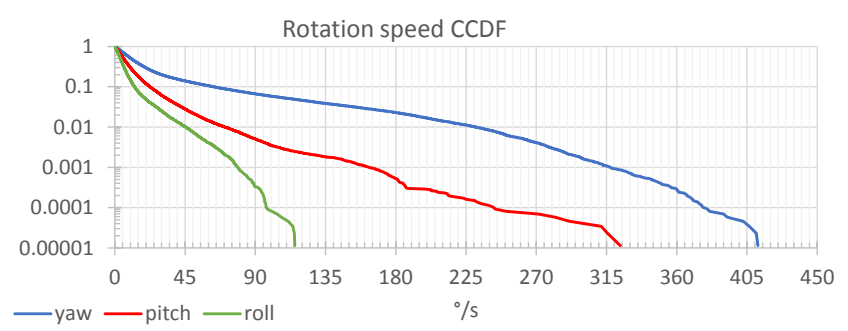

Figure 4: CCDF of rotation speeds during user test.

expressed by functions

$$
\begin{aligned}
\widehat{\mathrm{SSIM}} & =f(V, R) \\
\widehat{\mathrm{VMAF}} & =g(V, R)
\end{aligned}
$$

They formulate impacts of the key parameters and related trends. Furthermore, they might be inverted in order to express requirements on $R$ (or $V$ ) for given SSIM or VMAF values. The fitting functions (1) and (2) are calculated using the MatLab function NonLinearModel.fit.

\section{RESULTS}

To determine the lower and upper bounds for bitrates, two types of experiments were conducted with various settings. One of which we refer to as Continuous rotation and the other as Twitch recovery delay. The former takes ten samples of instantaneous SSIM and $\mathrm{VMAF}$ within a constant rotation at various bitrates. The latter computes the SSIM and VMAF of each frame where a quick rotation is applied to the camera for a brief period of frames. The quick rotation emulates a user making a twitch head-movement, its purpose is to determine how long the reduced quality caused by such rotations will persist.

Initially in this chapter, we determine the lower and upper bitrate limits by using purely artificial rotations in the continuous rotation and twitch recovery delay experiments. These limits are thereafter used in tests with a recording of real human head movements for verification.

\subsection{Continuous Rotation}

Results from taking ten samples of instantaneous SSIM and VMAF at various bitrates and head-rotation speeds for a common HMD and mobile phone resolution are shown in Figure 5. As a common HMD resolution, we use 1080×1200@90, which is used in the HTC Vive and Oculus Rift. As a mobile phone resolution, we use 1280×1440@60. 

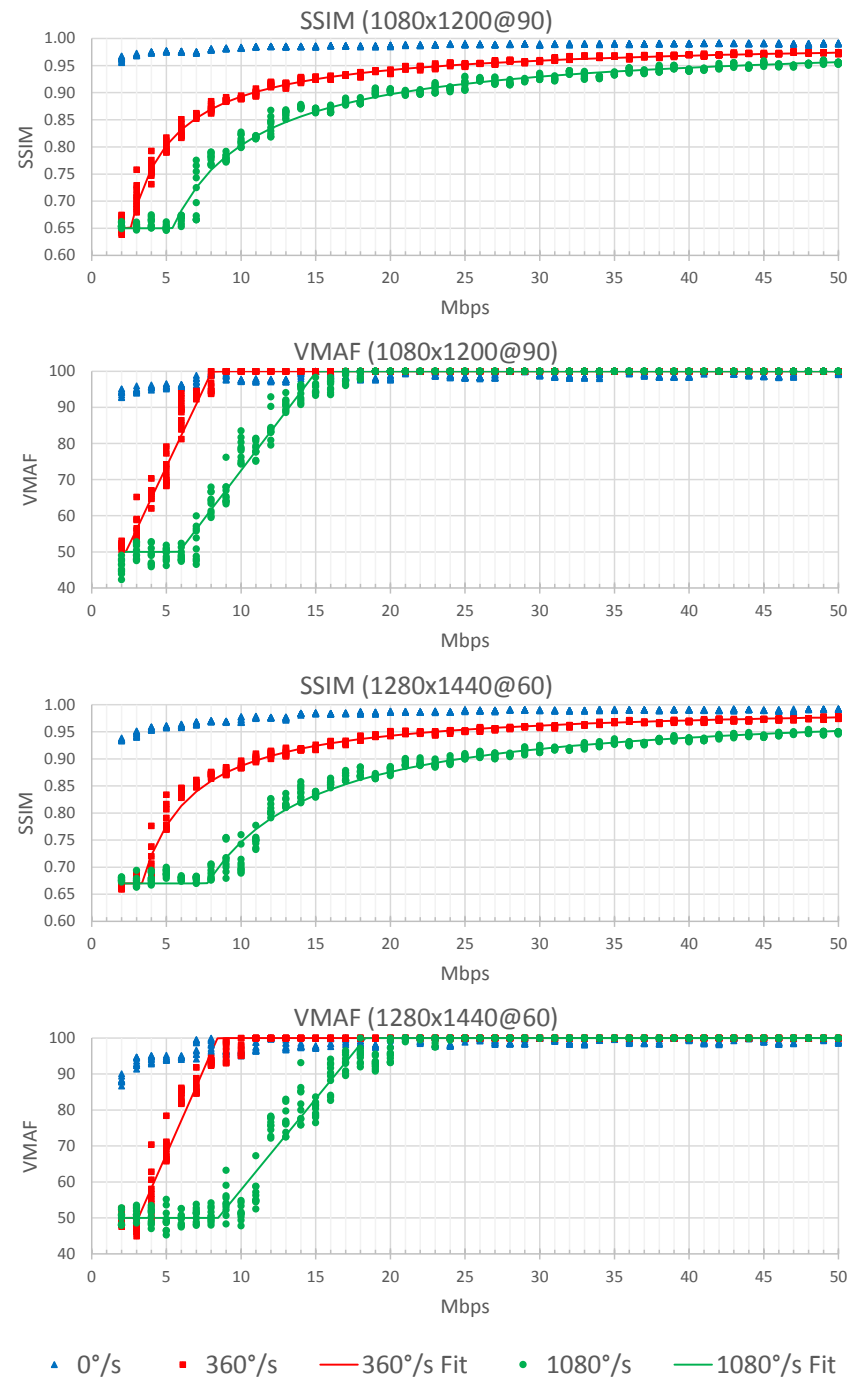

Figure 5: SSIM and VMAF at various bitrates and rotation speeds for a common HMD resolution (top) and mobile phone resolution (bottom). 10 samples were recorded for each bitrate.

Table 2: Coefficients and performance of the SSIM-related fits.

\begin{tabular}{|c|c|c|c|c|c|c|c|}
\hline & $b_{0}$ & $b_{1}$ & $b_{2}$ & $b_{3}$ & $b_{4}$ & $\mathcal{R}^{2}$ & RMSE \\
\hline HMD & 0.650 & 0.368 & $1.23 \mathrm{e}-3$ & 0.850 & $7.90 \mathrm{e}-5$ & 0.991 & 0.008 \\
\hline Mobile & 0.670 & 0.268 & $2.26 \mathrm{e}-3$ & 0.957 & $6.69 \mathrm{e}-5$ & 0.988 & 0.010 \\
\hline
\end{tabular}

4.1.1 Trends. The measured SSIM values are shown in Figure 5, together with the fits for $360^{\circ} / \mathrm{s} \leq V \leq 1080^{\circ}$ s given by

$$
f(V, R)=\max \left\{b_{0}, 1-\left(b_{1}+b_{2} V\right) R^{-\left(b_{3}+b_{4} V\right)}\right\}
$$

with coefficients $b_{i}$, coefficients of determination $\mathcal{R}^{2}$ and root mean squared error (RMSE) as shown in Table 2. The SSIM values ap-
Table 3: Coefficients and performance of the VMAF-related fits.

\begin{tabular}{|c|c|c|c|c|c|c|}
\hline & $b_{1}$ & $b_{2}$ & $b_{3}$ & $b_{4}$ & $\mathcal{R}^{2}$ & RMSE \\
\hline HMD & 36.13 & $-1.66 \mathrm{e}-2$ & 10.31 & $-4.52 \mathrm{e}-3$ & 0.986 & 1.78 \\
\hline Mobile & 27.23 & $-1.86 \mathrm{e}-2$ & 11.62 & $-6.07 \mathrm{e}-3$ & 0.980 & 2.38 \\
\hline
\end{tabular}

proach the optimal value 1 over $R$ in an asymptotic way from below, slower as $V$ grows, and faster in the HMD case.

Also, the measured VMAF values are shown in Figure 5, together with the corresponding fits for $360 \% \leq V \leq 1080 \% \mathrm{~s}$

$$
g(V, R)=\max \left\{50, \min \left\{100, b_{1}+b_{2} V+b_{3} R+b_{4} V R\right\}\right\}
$$

Obviously, the latter differ in shape from their SSIM counterparts; they are well-approximated by linear functions in both $V$ and $R$, limited between VMAF $=50$ and 100, with coefficients reported in Table 3. The negative signs of the coefficients $b_{2}$ and $b_{4}$ reveal that an increased value of $V$ slows down the approach towards the maximal value of 100 as $R$ grows. As for SSIM, the gradient is steeper in the HMD case. However, the VMAF values rise earlier and steeper over $R$ from their minimal values, and finally reach their top value 100 , in contrary to the asymptotic behaviour of SSIM.

For both SSIM and VMAF, the curves for $0 \% \mathrm{~s}$, which are not covered by above models (3) and (4), start from rather high scores, increase slightly and thereafter remain quite constant. This means that a high quality can be maintained with rather low bitrates if the camera does not move.

4.1.2 Recommendations. Depending on the requirements on rotation speed, i.e. how fast the users are expected to rotate their heads, and where the line for required SSIM and/or VMAF is drawn, one may use the results to determine the required bitrate. An application that requires support for fast head rotations should clearly use a higher bitrate than one that does not.

Solving the SSIM-related equation (3) for $R$ gives

$$
\tilde{R}=\left(\frac{1-\mathrm{SSIM}}{b_{1}+b_{2} V}\right)^{-1 /\left(b_{3}+b_{4} V\right)}
$$

with coefficients according to Table 2 . As an example, suppose a minimum SSIM value of 0.9 is desired at all times. Equation 5 then indicates bitrate requirements of $10.8 \mathrm{Mbps}$ for $\mathrm{HMD}$ and $11.3 \mathrm{Mbps}$ for mobile with $V=360 \%$, as well as $20.6 \mathrm{Mbps}$ for HMD and $24.7 \mathrm{Mbps}$ for mobile with $\mathrm{V}=1080^{\circ} / \mathrm{s}$, respectively.

In the VMAF case, Equation (4) leads to

$$
\tilde{R}=\frac{\mathrm{VMAF}-b_{1}-b_{2} V}{b_{3}+b_{4} V}
$$

For $\mathrm{VMAF}=100$, the model estimates bitrate requirements of 8.1Mbps for HMD and 8.4Mbps for mobile with $V=360^{\circ} / \mathrm{s}$, as well as $15.1 \mathrm{Mbps}$ for HMD and $18.4 \mathrm{Mbps}$ for mobile with $\mathrm{V}=1080^{\circ} \mathrm{s}$, respectively. Note that these numbers give no room for deviations though, and should be used with a safety margin of a few Mbps.

According to the empirical data, in the example case of SSIM = 0.9 , if head-movements of up to $360 \% \mathrm{~s}$ can be expected as maximum, we require $12 \mathrm{Mbps}$ per eye in HMD resolutions and $13 \mathrm{Mbps}$ on mobile to be on the safe side. These are the lowest bitrates with 
an SSIM higher than 0.9 according to Figure 5. For $1080^{\circ} / \mathrm{s}, 24$ and $28 \mathrm{Mbps}$ are required to maintain the same SSIM, respectively.

To set our recommended lower and upper bitrate limits while being on the safe side, we look for the lowest bitrate where all VMAF scores are 100 . For the lower-limit speed of $360^{\circ} / \mathrm{s}$, this bitrate is $9 \mathrm{Mbps}$ for HMD and 11Mbps for mobile. For the upper limit $1080^{\circ} \mathrm{s}$, it is 19 and $21 \mathrm{Mbps}$, respectively. To conclude, the results of these tests imply the following requirements per eye:

(1) HMD, 1080×1200@90: 9-19Mbps

(2) Mobile, 1280×1440@60: 11-21Mbps

\subsection{Twitch Recovery Delay}

Overall, a poor instantaneous quality-score within a continuous and quick rotation, like those seen in the previous section, may not necessarily indicate that the used bitrate is too low. It is likely that the reduced quality during such movements may very well go unnoticed due to the scene literally flashing past the eyes of the user. Naturally, this depends on the speed of the rotation, a higher speed means a lower likelihood of the user noticing any quality reduction. Determining at what speeds the user may notice quality reductions and to what degree is outside the scope of this work and thus left for future studies. Regardless, we may assume that quality loss within movements are not catastrophic if the movement is quick enough, however, the loss may hurt the experience if it remains after the movement stops. To determine for how long the quality loss, in terms of SSIM and VMAF, remains after a motion has finished, we capture 300 successive frames where a rotation of $1080 \%$ is applied to the camera temporarily and abruptly stopped. The rotation speed $1080^{\circ} \mathrm{s}$ is applied at frame 20 and set back to zero at frame 40 , thus a twitch motion of 20 frames is emulated, see Figure 6. Results show that such a twitching rotation will cause a quality decrease that lasts for several frames following the motion stop. Higher bitrates will maintain a higher quality within the motion, and will also more quickly repair the damage. To save space, we show only the graphs produced by tests with the HMD resolution, the ones for mobile are very similar.

Although the scores take time to recover, we may assume that most humans are unable to distinguish the loss when the SSIM or VMAF reaches a sufficiently high score. We therefore present the exact number of frames taken from motion stop to recovery in terms of a few high SSIM and VMAF limits for all tested bitrates, we do this for both resolutions, see Table 4 and 5 . In both tables, the bitrates 4 and 6Mbps never reach back to an SSIM of 0.98 nor VMAF of 100 in the recorded time frame, while all other bitrates eventually do. We also note that VMAF generally settles at $10 \mathrm{Mbps}$ in both resolutions, without much improvement at higher bitrates. In terms of VMAF, a large decrease in delay is seen when going from $8 \mathrm{Mbps}$ to $10 \mathrm{Mbps}$, followed by very similar delays up to the maximum recorded bitrate of $32 \mathrm{Mbps}$. The main purpose of the tables is to see where the minimum bitrate limit should be drawn, i.e. where we start to see a significant drop-off in cost-efficiency when compared to higher bitrates. If we consider the VMAF model to be a good estimator, we reach that limit around $10 \mathrm{Mbps}$ in both cases. SSIM reaches no such limit though, but will gradually decrease its delay with increased bitrate. At higher SSIM, the decrease in delay due to increase in bitrate remains high between the recorded bitrates. For
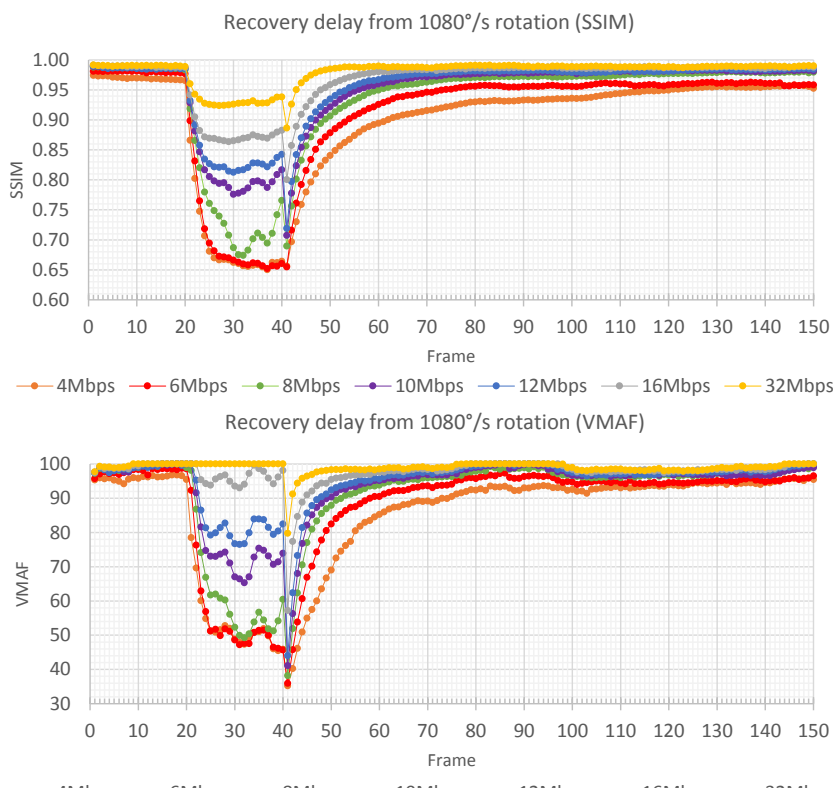

Figure 6: Impact and time required to repair quality loss caused by twitch motion at various bitrates. The motion consists of a rotation at $1080 \%$ s that abruptly begins at frame 20 and stops at frame 40.

example, with the mobile resolution at an SSIM of 0.98 , in Table 5, we see approximately the numbers: $>260,>260,200,100,50,20$ and 5 . Note though that the increase in bitrate between the levels are 2, $2,2,2,2,4$ and 16 respectively. So there is a drop-off in efficiency at higher bitrates, but it is not as pronounced as in the VMAF-case.

The results from these tests pose the following questions: For how many frames may we have a reduced quality, and what is the lowest acceptable temporary quality according to users? If we assume that an SSIM of 0.98 and VMAF of 100 are the required limits, we need at least $8 \mathrm{Mbps}$ due to lower bitrates never reaching these levels in the measured time. Furthermore, due to the significantly higher VMAF score and cost-efficiency drop-off in VMAF beyond $10 \mathrm{Mbps}$, we recommend $10 \mathrm{Mbps}$ as the minimum bitrate in both cases. For the overall recommendations, we therefore update the minimum HMD bitrate from $9 \mathrm{Mbps}$ to $10 \mathrm{Mbps}$ to be on the safe side. For the mobile case, we stay with $11 \mathrm{Mbps}$ as recommendation and do not downgrade it to $10 \mathrm{Mbps}$.

\subsection{Realistic Rotations}

So far, we have only shown the objective quality scores when fixed rotations have been applied to the camera, which is sufficient to cover edge cases, but is not a realistic use case. Since the user test presented in Section 3.2.3 has given us realistic human head movements, we may apply these to the streaming prototype and see how realistic movements affect the quality scores. It is not practical to use the entire recording since it consists of above 80000 frames. We therefore take a small sample of 4500 frames ( 50 seconds) between frames 60500-65000 of the recording shown in Figure 3. This interval contains several rotation-speed peaks which is shown 
Table 4: Twitch Recovery Delay $(1080 \times 1200 @ 90)$ (Number of frames until one sample scores above limit)

\begin{tabular}{|c|c|c|c|c|c|c|c|}
\hline & \multicolumn{7}{|c|}{ Bitrate (Mbps) } \\
\hline SSIM & 4 & 6 & 8 & 10 & 12 & 16 & 32 \\
\hline$\geq 0.90$ & 22 & 14 & 9 & 8 & 6 & 4 & 2 \\
\hline$\geq 0.92$ & 34 & 19 & 13 & 10 & 8 & 5 & 2 \\
\hline$\geq \mathbf{0 . 9 4}$ & 66 & 26 & 17 & 14 & 11 & 7 & 3 \\
\hline$\geq 0.96$ & 154 & 66 & 28 & 20 & 16 & 11 & 4 \\
\hline$\geq 0.98$ & $>260$ & $>260$ & 94 & 66 & 36 & 20 & 8 \\
\hline VMAF & & & & & & & \\
\hline$\geq 80$ & 15 & 9 & 6 & 5 & 4 & 3 & 2 \\
\hline$\geq 85$ & 20 & 12 & 8 & 6 & 5 & 4 & 2 \\
\hline$\geq 90$ & 35 & 19 & 13 & 10 & 8 & 5 & 2 \\
\hline$\geq 95$ & 103 & 36 & 23 & 18 & 16 & 10 & 4 \\
\hline$\geq 100$ & $>260$ & $>260$ & 178 & 45 & 44 & 39 & 37 \\
\hline
\end{tabular}

Table 5: Twitch Recovery Delay (1280×1440@60) (Number of frames until one sample scores above limit)

\begin{tabular}{|c|c|c|c|c|c|c|c|}
\hline & \multicolumn{7}{|c|}{ Bitrate (Mbps) } \\
\hline SSIM & 4 & 6 & 8 & 10 & 12 & 16 & 32 \\
\hline$\geq 0.90$ & 16 & 10 & 7 & 5 & 3 & 2 & 0 \\
\hline$\geq 0.92$ & 25 & 14 & 10 & 7 & 5 & 3 & 0 \\
\hline$\geq 0.94$ & 54 & 23 & 13 & 10 & 7 & 4 & 1 \\
\hline$\geq 0.96$ & $>260$ & 51 & 22 & 15 & 13 & 7 & 2 \\
\hline$\geq 0.98$ & $>260$ & $>260$ & 196 & 105 & 50 & 20 & 5 \\
\hline VMAF & & & & & & & \\
\hline$\geq 80$ & 12 & 7 & 5 & 3 & 2 & 1 & 0 \\
\hline$\geq 85$ & 16 & 10 & 6 & 4 & 3 & 2 & 0 \\
\hline$\geq 90$ & 25 & 15 & 10 & 7 & 5 & 3 & 1 \\
\hline$\geq 95$ & 73 & 26 & 18 & 15 & 12 & 7 & 2 \\
\hline$\geq \mathbf{1 0 0}$ & $>260$ & $>260$ & 74 & 28 & 28 & 25 & 25 \\
\hline
\end{tabular}

in the left graph in Figure 7. The recording contains the yaw, pitch and roll of the camera of each frame. Starting at frame 60000, we simply apply the rotations to the camera in the prototype game each frame while streaming and saving to PNG. Note that we start at 60000 and cut out 500 frames to let the codec get into a steady state, this is applied to all recordings throughout this work, but happens to be more noticeable in this case.

Figure 7 shows how the objective quality scores vary when realistic user rotations are applied to the prototype 3D scene. This test was conducted with the HMD resolution two times, once for $10 \mathrm{Mbps}$ and once for $19 \mathrm{Mbps}$, since these are the recommended lower and upper bitrates determined by previous tests in Sections 4.1 and 4.2. We note from the results that far from every frame stays at a VMAF of exactly 100, though most do and all come close. Also, there is clearly an increase in quality in both VMAF and SSIM when increasing the bitrate to $19 \mathrm{Mbps}$. The increase does not seem very cost-efficient though, considering the bitrate is almost doubled for a relatively small increase in scores, especially in terms of VMAF. In detail, results show that an SSIM of $0.91-0.98$ with an average of 0.95 is achieved at $10 \mathrm{Mbps}$. At $19 \mathrm{Mbps}$, the range is $0.95-0.99$ with an average of 0.97 . VMAF for $10 \mathrm{Mbps}$ ranges between $91-100$ with an average of 99 . For $19 \mathrm{Mbps}$, the range is $96-100$ with an

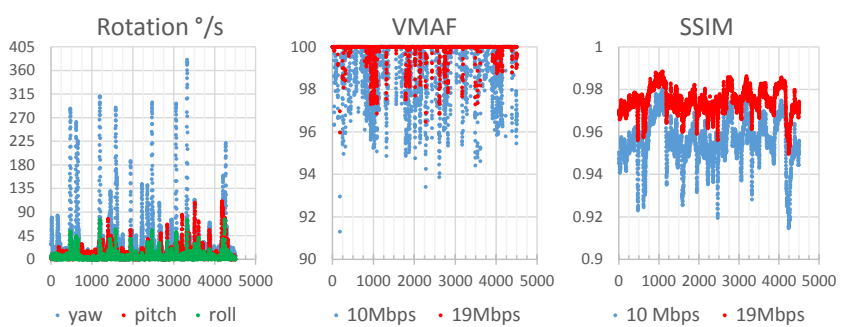

Figure 7: Example of VMAF and SSIM of 4500 frames when recorded rotations from the user test were used with the streaming prototype and $3 D$ scene.

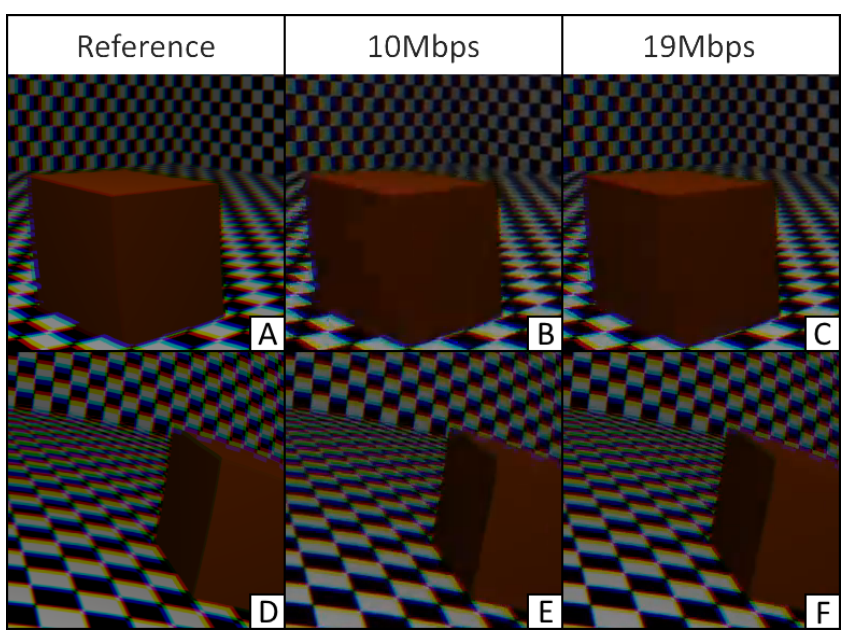

Figure 8: $256 \times 256$ samples from the image with the lowest SSIM (top) and highest (bottom) at 10Mbps.

SSIM: $\mathrm{B}=0.91, \mathrm{C}=0.95, \mathrm{E}=0.98, \mathrm{~F}=0.99$.

VMAF: $\mathrm{B}=100, \mathrm{C}=100, \mathrm{E}=97, \mathrm{~F}=98$.

average of 100. In conclusion, the recommended bitrates produce high scores also with the realistic head movements used in this test.

As a reference for the reader, we provide samples of the images with the best and worst SSIM of this test according to the 10Mbps recording. These are shown in Figure 8. Note here also how SSIM and VMAF are not always in agreement, the image with the worst SSIM still had a VMAF of 100, and the one with the best SSIM had a VMAF slightly less than 100 for both bitrates.

\section{CONCLUSIONS}

We have presented recommendations of bitrates in VR-streaming at a per-eye resolution common in HMDs, 1080×1200@90 fps, and in mobile phones, $1280 \times 1440 @ 60 \mathrm{fps}$. Requirements were calculated using SSIM and VMAF by using an in-house prototype with NVENC and NVDEC. The prototype encodes and decodes two NV12 H.264-images in parallel in separate streams, which is important to consider if using the recommendations of this work. According to our results, we would recommend using a bitrate range of 10$19 \mathrm{Mbps}$ per eye for the HMD resolution, and $11-21 \mathrm{Mbps}$ for mobile, which results in a total of $20-38 \mathrm{Mbps}$ and $22-42 \mathrm{Mbps}$. The lower 
limits should produce an excellent quality in most scenarios, and are followed by large drop-offs in cost-efficiency. Bitrate increases above the upper limits are very inefficient and would largely be a waste according to our results.

The recommendations are entirely based on VMAF. Lower bitrates are based on drop-offs in cost-efficiency for VMAF and on the assumption that an application should at least support $360 \% \mathrm{~s}$ rotations without quality loss at constant rotation. Upper bitrates are also based on VMAF and the assumption that human heads will rotate no faster than $1080^{\circ} / \mathrm{s}$.

\section{FUTURE WORK}

We assume that humans may rotate their heads and thus VR HMDs at a speed between $0-1080^{\circ} / \mathrm{s}$. In future studies, we may with the help of real users determine a probability distribution of required rotation speeds. These speeds will be application dependent, but it may be possible to determine genre-specific rotation requirements for example.

Thus far, we have used objective measurements to estimate the bitrate requirements. Our results are largely based on a model of VMAF that was not developed for VR, but has shown promise in related work on 360 VR [13]. In future work, we may involve humans in the research in order to get subjective quality measures and more definitive results that are confirmed by human users. This work may serve as a guide to such studies, perhaps most importantly, we provide a range of bitrates that may be used in further testing.

We have only studied two resolutions and framerates and can therefore not say much about how these parameters affect bitrate. Future work may involve determining the effects of resolution and framerate changes in detail.

\section{ACKNOWLEDGMENTS}

This work was supported in part by the KK Foundation, Sweden, through the project "ViaTecH" under contract number 20170056. The authors would like to thank our colleagues in the ViaTecH project for the feedback and discussions.

\section{REFERENCES}

[1] Eduardo Cuervo, Alec Wolman, Landon P. Cox, Kiron Lebeck, Ali Razeen, Stefan Saroiu, and Madanlal Musuvathi. 2015. Kahawai: High-Quality Mobile Gaming Using GPU Offload. In Proceedings of the 13th Annual International Conference on Mobile Systems, Applications, and Services (Florence, Italy) (MobiSys '15). Association for Computing Machinery, New York, NY, USA, 121-135. https://doi.org/10.1145/2742647.2742657

[2] FFmpeg. 2016. FFmpeg - A complete, cross-platform solution to record, convert and stream audio and video. Retrieved April 8, 2020 from https://ffmpeg.org

[3] FFmpeg. 2020. Calculating SSIM with FFmpeg. Retrieved April 17, 2020 from https://ffmpeg.org/ffmpeg-all.html\#ssim
[4] Philippe Fuchs. March, 2019. Virtual Reality Headsets - A Theoretical and Pragmatic Approach. Cambridge University Press, University of Oulu, Finland. https: //books.google.se/books?id=lfPJDwAAQBAJ

[5] Steve Göring, Janto Skowronek, and Alexander Raake. 2018. DeViQ - A deep no reference video quality model. In Human Vision and Electronic Imaging (Northwestern University, USA) (HVEI '18). Ingenta Connect, Oxford, UK, 16. https://doi.org/10.2352/ISSN.2470-1173.2018.14.HVEI-518

[6] Gazi Karam Illahi, Thomas Van Gemert, Matti Siekkinen, Enrico Masala, Antti Oulasvirta, and Antti Ylä-Jääski. 2020. Cloud Gaming with Foveated Video Encoding. ACM Trans. Multimedia Comput. Commun. Appl. 16, 1, Article 7 (Feb. 2020), 24 pages. https://doi.org/10.1145/3369110

[7] International Telecommunication Union (ITU). 2008. P.910 : Subjective video quality assessment methods for multimedia applications. Retrieved April 14, 2020 from https://www.itu.int/rec/T-REC-P.910-200804-I

[8] Zeqi Lai, Y. Charlie Hu, Yong Cui, Linhui Sun, and Ningwei Dai. 2017. Furion: Engineering High-Quality Immersive Virtual Reality on Today's Mobile Devices. In Proceedings of the 23rd Annual International Conference on Mobile Computing and Networking (Snowbird, Utah, USA) (MobiCom '17). Association for Computing Machinery, New York, NY, USA, 409-421. https://doi.org/10.1145/3117811.3117815

[9] Luyang Liu, Ruiguang Zhong, Wuyang Zhang, Yunxin Liu, Jiansong Zhang, Lintao Zhang, and Marco Gruteser. 2018. Cutting the Cord: Designing a High-quality Untethered VR System with Low Latency Remote Rendering. In Proceedings of the 16th Annual International Conference on Mobile Systems, Applications, and Services (Munich, Germany) (MobiSys '18). ACM, New York, NY, USA, 68-80. https://doi.org/10.1145/3210240.3210313

[10] Netflix. 2014. VMAF - Video Multi-Method Assessment Fusion. Retrieved April 8, 2020 from https://github.com/Netflix/vmaf

[11] Netflix. 2018. Predict Quality on a 1080p HDTV screen at 3H. Retrieved April 20, 2020 from https://github.com/Netflix/vmaf/blob/master/resource/doc/models.md

[12] Nvidia. 2013. Nvidia Video Codec SDK. Retrieved January 16, 2020 from https: //developer.nvidia.com/nvidia-video-codec-sdk

[13] Marta Orduna, César Díaz, Lara Muñoz, Pablo Pérez, Ignacio Benito, and Narciso García. 2020. Video Multimethod Assessment Fusion (VMAF) on 360VR Contents. IEEE Transactions on Consumer Electronics 66, 1 (Feb. 2020), 22-31. https://doi. org/10.1109/TCE.2019.2957987

[14] Ulrik Röijezon, Mats Djupsjöbacka, Martin Björklund, Charlotte Häger-Ross, Helena Grip, and Dario G. Liebermann. 2010. Kinematics of fast cervical rotations in persons with chronic neck pain: a cross-sectional and reliability study. BMC Musculoskeletal Disorders 11, 222, Article 222 (Sept. 2010). https://doi.org/10. 1186/1471-2474-11-222

[15] Ashutosh Singla, Stephan Fremerey, Werner Robitza, Pierre Lebreton, and Alexander Raake. 2017. Comparison of Subjective Quality Evaluation for HEVC Encoded Omnidirectional Videos at Different Bit-Rates for UHD and FHD Resolution. In Proceedings of the on Thematic Workshops of ACM Multimedia 2017 (Mountain View, California, USA) (Thematic Workshops '17). Association for Computing Machinery, New York, NY, USA, 511-519. https://doi.org/10.1145/3126686.3126768

[16] Ivan Slivar, Mirko Suznjevic, and Lea Skorin-Kapov. 2018. Game Categorization for Deriving QoE-Driven Video Encoding Configuration Strategies for Cloud Gaming. ACM Trans. Multimedia Comput. Commun. Appl. 14, 3s, Article 56 (June 2018), 24 pages. https://doi.org/10.1145/3132041

[17] TPCAST. 2017. Wireless Adapter for VIVE. Retrieved April 15, 2020 from https: //www.tpcast.cn/index.php?s=/Front/Goods/index/good/10228/1/en-us

[18] Valve. 2015. OpenVR SDK. Retrieved January 16, 2020 from https://github.com/ ValveSoftware/openvr

[19] Valve. 2016. The Lab. Retrieved May 5, 2020 from https://store.steampowered. com/app/450390/The_Lab/

[20] Ruiguang Zhong, Manni Wang, Zijian Chen, Luyang Liu, Yunxin Liu, Jiansong Zhang, Lintao Zhang, and Thomas Moscibroda. 2017. On Building a Programmable Wireless High-Quality Virtual Reality System Using Commodity Hardware. In Proceedings of the 8th Asia-Pacific Workshop on Systems (Mumbai, India) (APSys '17). ACM, New York, NY, USA, Article 7, 7 pages. https: //doi.org/10.1145/3124680.3124723

[21] Zhou Wang, A. C. Bovik, H. R. Sheikh, and E. P. Simoncelli. 2004. Image quality assessment: from error visibility to structural similarity. IEEE Transactions on Image Processing 13, 4 (April 2004), 600-612. https://doi.org/10.1109/TIP.2003. 819861 